\title{
VALIDACIÓN DE UN MÉTODO PARA CUANTIFICACIÓN DE ACETAMINOFÉN EN TABLETAS DE 500 MG POR ESPECTROFOTOMETRÍA ULTRAVIOLETA PARA LA PRUEBA DE UNIFORMIDAD DE CONTENIDO
} VALIDATION OF A METHOD FOR QUANTIFICATION ACETAMINOPHEN 500 MG TABLETS IN ULTRAVIOLET SPECTROPHOTOMETRY FOR TESTING OF CONTENT UNIFORMITY

\author{
Esteban Pérez López * $\quad$ Alfonso Rojas Hernández ${ }^{\dagger}$ \\ Recibido: 18 de diciembre del 2015 \\ Aprobado: 20 de mayo del 2016 \\ DOI: http://dx.doi.org/10.15517/isucr.v17i35.25563
}

\section{Resumen}

El presente artículo contiene los detalles de la validación del método analítico para la cuantificación de acetaminofén, en este caso para aplicar a la prueba de uniformidad de contenido a tabletas de $500 \mathrm{mg}$, por la técnica de espectroscopia ultravioleta a una longitud de onda de trabajo de $242 \mathrm{~nm}$, empleando como disolvente ácido clorhídrico a una concentración de 0,1 M. Los parámetros evaluados fueron la linealidad, exactitud, repetitividad y precisión intermedia, siguiendo estrictamente la guía y política de validación del Ente Costarricense de Acreditación (ECA), para un método normalizado modificado.

\footnotetext{
*Máster en Sistemas Modernos de Manufactura y Bach. en Laboratorista Químico. Docente UCR-Recinto Grecia. esteban.perezlopez@ucr.ac.cr

†Estudiante de Bach. en Laboratorista Químico. UCR. alfonso.rojas@ucr.ac.cr
} 
Todos los parámetros evaluados arrojaron resultados satisfactorios por lo que se considera que el método es lineal, preciso y exacto para el fin que se desarrolló; en este caso para la prueba de uniformidad de contenido en su primera etapa donde la especificación es que el valor de aceptación (AV) calculado no sea mayor a L1 = 15, analizando 10 dosis individualmente según la USP 38 .

Palabras clave: Validación de métodos; control de calidad; espectrofotometría ultravioleta; acetaminofén; paracetamol.

\begin{abstract}
This article contains the details of the validation of the analytical method for the quantification of acetaminophen, in this case to apply to the uniformity test of content to tablets of $500 \mathrm{mg}$, by the technique of UV spectroscopy at a wavelength of work $242 \mathrm{~nm}$, using hydrochloric acid as solvent at a concentration of $0.1 \mathrm{M}$. the parameters evaluated were linearity, accuracy, repeatability and intermediate precision, strictly following the guidance and validation policy Costa Rican Accreditation Entity (ECA) for a modified standardized method.
\end{abstract}

All evaluated parameters yielded satisfactory results so it is considered that the method is linear, precise and accurate for the purpose it was developed; in this case to test for content uniformity in its first stage where the specification is that the acceptance value (AV) calculated not greater than $\mathrm{L} 1=15,10$ doses analyzed individually according to USP 38 .

Keywords: Method validation; quality control; ultraviolet spectrophotometry; acetaminophen; paracetamol.

\section{Introducción}

Uno de los principales retos actuales, en cuanto al desarrollo de analgésicos es lograr un producto estable, homogéneo, efectivo y que sus propiedades se mantengan en el tiempo [Avila. P, Ferro. $V \&$ Tello. M. 1999]. Para determinar el cumplimiento de estas condiciones es que se emplean las pruebas físico-químicas de control de calidad de medicamentos; y con el fin de garantizar la eficiencia de las pruebas de control de calidad es que se hace necesario validar los métodos, cuando estos no son oficiales.

Las actividades de confirmación de la calidad para la OMS (1999) tienen como objetivo realizar prue- bas fácilmente aplicables y reproducibles con la menor cantidad de reactivos posibles, facilitando un medio para demostrar la estabilidad de degradación e identidad del producto farmacéutico.

Estas pruebas pueden ser prácticas básicas de calidad, las cuales no incluyen métodos cuantitativos sino solo de identidad, por otro lado las prácticas expuestas en las farmacopeas internacionales no son superadas por pruebas de identidad y es obligación de las empresas productoras realizar prácticas cuali-cuantitativas de los productos fármacos, con prácticas trazables y normalizadas (OMS, 1999).

Para [Prieto. Y. 2008] en la década de 1960 surge en la industria farmacéutica norteamericana el concepto 
de Buenas Prácticas de Fabricación (BPF), a partir de varios incidentes relacionados con la fabricación de medicamentos, que culminaron en serios perjuicios, incluso la muerte de pacientes. Con él se establece un modo de "hacer bien" o "de forma correcta" todos los procesos relacionados con la fabricación de los medicamentos, de manera que se evite la posible afectación de la identidad, la seguridad y la eficacia de los productos, lo cual podría constituir un riesgo para la salud de la población.

Las buenas prácticas de laboratorio (BPL) han logrado fomentar y corroborar que un medicamento cumpla con las especificaciones del fabricante. Esto por medio de métodos válidos de análisis, para garantizar la eficacia, seguridad y homogeneidad de un medicamento, con resultados fiables y documentados desde la práctica analítica [Lavaut, M \& Rodríguez, J. 2009].

Por lo que en conjunto las BPL, las farmacopeas y los métodos introducidos estandarizados, forman las bases para constituir un adecuado plan de calidad que asegure el cumplimiento de parámetros obligatorios, con respecto a estándares internacionales, logrando la homogeneidad de las actividades de manufactura.

Para [Lavaut, M \& Rodríguez, J. 2009], lo anterior indica que no solo se debe considerar aspectos físicos de calidad, sino también las formas analíticas de corroborar las propiedades del producto, siguiendo métodos adecuados, o adecuando nuevos métodos, según las restricciones de trabajo, pero que sean funcionales $y$ equivalentes en cuanto a resultados con los oficiales.

Según [Caridad. M, Garcia. P, Porto.] los métodos espectrofotométricos resultan de gran utilidad para el control de la calidad porque son rápidos, sencillos y económicos. Basado en esto, se determina la importancia de utilizar un método por espectrofotometría valida- do, con el fin de mejorar el tiempo de realización de las pruebas, de no emplear otras técnicas que conllevan mayor consumo de reactivos, horas analista, horas equipo y requieren mayor capacidad técnica del operador.

En este trabajo se presenta la validación de un método espectrofotométrico para la cuantificación de acetaminofén en la prueba de uniformidad de contenido aplicada a tabletas de $500 \mathrm{mg}$, comprendiendo solo el primer criterio de la prueba, en el que se analizan diez dosis individuales y cuya especificación es que el valor de aceptación (AV) calculado no sea mayor a L1 igual a 15 (L1 = 15) según establece la farmacopea oficial.

\section{Fundamento}

Para [Arias. T. 1999] el efecto de un fármaco está dado por el principio activo, el cual es una sustancia o mezcla de sustancias afines dotadas de un efecto farmacológico específico o que, sin poseer actividad, al ser administrados al organismo la adquieren luego que sufren cambios en su estructura química, como es el caso de los profármacos.

En el caso específico de la acetaminofén, el cual es uno de los analgésicos de más amplio uso en el mundo, su buen perfil de seguridad, eficacia y el hecho de ser un medicamento de venta libre, hacen que la formulación y la utilización de este fármaco sea muy común, tanto en la población adulta como en la pediátrica [Fernández. D, Fernández. D \& Mancipe. L. 2010].

Según [Hendrickson R.G. 2015] la acetaminofén o paracetamol, es un analgésico y antipirético no esteroideo, cuyo mecanismo de acción es único, inhibiendo la enzima ciclooxigenaza y prostaglandinas responsables de la aparición del dolor. Debido a su efectividad y gran 
consumo de la población, la acetaminofén es un medicamento foco de constante investigación y del cual se debe velar por su calidad y monitoreo.

Según las Buenas Prácticas de la OMS (2010), el control de calidad de productos farmacéuticos son por lo general análisis repetitivos de muestras de ingredientes farmacéuticos activos o de un número limitado de productos farmacéuticos, por lo tanto es asegurar que las materias primas, productos intermedios, materiales de envase y productos farmacéuticos terminados cumplan con las especificaciones establecidas para identidad, contenido, pureza y otras características.

La seguridad de que un producto cumple con las especificaciones, sólo es relevante si los métodos a través de los cuales se llega a los resultados, son oficiales o están validados. De aquí la importancia de contar con procedimientos validados, los cuales según [Durán, D. 2011] se define como la acción en la que se prueba que cualquier material, proceso, procedimiento, actividad, equipo o mecanismo empleado en la fabricación o control, logrará los resultados para los cuales se destina. La obtención y documentación de datos demostrativos de la confiabilidad de un método debe producir el resultado esperado dentro de los límites definidos.

Con lo que respecta a la metodología para el análisis cuantitativo de acetaminofén (determinación de contenido y uniformidad de contenido), la prueba oficial aplicable a las tabletas según la Farmacopea de los Estados Unidos (USP 38) es por la técnica de cromatografía líquida de alta resolución [Delfino. R, Radovancich. D \& Sarno. M. 2006].

En lo que respecta a la prueba de uniformidad, esta se describe como el examen para determinar la variabilidad de la cantidad del principio activo presente en las unidades que sirven de muestra de un determinado lote. Se recomienda aplicar esta prueba a las formas farmacéuticas que contienen una cantidad relativamente pequeña de un principio activo sumamente potente [Arias. T. 1999]. Aunque según la farmacopea de los Estados Unidos puede aplicarse en todos los casos.

La validación del método para la uniformidad, en este caso, se realiza por medio de la técnica de espectrofotometría ultravioleta, por las ventajas que esta técnica tiene. Para reseñar la técnica espectrofotométrica y particularmente la absorción en la región ultravioleta, esta utiliza radiación del espectro electromagnético comprendida entre 100 y los $300 \mathrm{~nm}$ que provoca transiciones electrónicas entre los orbitales atómicos, lo que provoca la excitación de electrones de valencia, y como consecuencia se pueden identificar espectros donde la longitud de onda de los picos se puede relacionar con determinados enlaces. Para generar el espectro se emplea un espectrofotómetro el cual genera un barrido espectral, que es empleado para identificar sustancias y su posterior cuantificación [Skoog, Holler \& Nieman. 2001].

Lo anterior es la base del método de espectrofotometría ultravioleta que se utiliza en este estudio, sin embargo, para asegurar que genera resultados fidedignos se debe realizar su adecuada validación la cual se realizó basándose en la política y guía de validación del ECA (2012). Según esta guía, el procedimiento a validar se encuentra dentro de la clasificación de "situación normalizada modificada", es decir, parte de un método ya estandarizado el cual ha sufrido modificaciones para ser aplicable al fin que se desea y para el cual se debe evaluar: linealidad, precisión, precisión intermedia y exactitud. En este caso, es importante reseñar que hay metodologías de cuantificación de acetaminofén por espectrofotometría ultravioleta en diferentes farmacopeas, pero en todos los casos difieren en condiciones con respecto a las 
que en este estudio se implementaron y validaron, por conveniencia analítica del laboratorio, además de que en algunos casos son métodos aplicados para otras pruebas y no referidas a la prueba de uniformidad de contenido. Asimismo según USP 38, la prueba del ensayo para acetaminofén en tabletas, se determina por Cromatografía de Líquidos de Alta Resolución (HPLC).

Teniendo claro lo anterior, con respecto a los parámetros a validar y sus respectivas definiciones, según la política de validación se define linealidad como la capacidad de un método de producir resultados que sean directamente, o por medio de una transformación matemática definida, proporcionales a la concentración de analito en la muestra. El término linealidad aplicado a un método analítico, se refiere al tramo de concentraciones del analito en el que la respuesta del sistema de medición es una función lineal de la concentración; la representación gráfica de este tramo (concentraciones frente a respuestas) debe exhibir una buena correlación de los puntos experimentales a la recta de regresión para que el método analítico en cuestión sea aceptable.

En lo referente a la precisión, el parámetro de repetibilidad es el grado de concordancia entre datos obtenidos aplicando un mismo procedimiento, sobre una misma muestra, con el mismo operador, en intervalos cortos de tiempo, utilizando el mismo equipamiento, dentro de un mismo laboratorio, es decir bajo las mismas condiciones. Por su parte, precisión intermedia es la obtenida aplicando un mismo procedimiento, sobre una misma muestra, en el mismo laboratorio, bajo condiciones diferentes de operación (equipo, analista, tiempo). Por último, la exactitud se define como grado de concordancia entre el resultado de una medición y un valor verdadero convencional, o un valor de referencia y el valor encontrado.

\section{Metodología}

El estudio consistió en la validación de un método espectrofotométrico para la cuantificación de acetaminofén en la prueba de uniformidad de contenido a tabletas de 500 $\mathrm{mg}$, y forma parte de una etapa previa del proyecto de investigación 540-B5-028 de la Universidad de Costa Rica, Sede de Occidente. Para la validación se utilizaron muestras de un solo lote de tabletas distribuidas por la CCSS y fabricadas en China. Las pruebas experimentales de parámetros de validación se ejecutaron entre agosto y noviembre del 2015 en el laboratorio de química del Recinto de Grecia de la UCR.

Para la validación del método, primeramente se evaluó la linealidad del método. Experimentalmente se realizó evaluando cinco niveles de concentración correspondientes al 80, 90, 100, 110 y $120 \%$ de la concentración normal de trabajo del método. Para esto se morterizaron finamente diez tabletas, pesadas independientemente en balanza analítica, luego se realizó la pesada del polvo por triplicado y correspondientes a cada porcentaje en balones de $100 \mathrm{ml}$. Se llenaron a $3 / 4$ partes con $\mathrm{HCl}$ 0.1 M y se colocan en baño ultrasónico por 10 minutos, hasta lograr la disolución total del principio activo. Luego se enfrían los balones con agua fría y se aforan con el mismo disolvente, se filtra cada uno con papel de filtro cuantitativo número 2, para eliminar los excipientes insolubles. Posteriormente se toma una alícuota de $1 \mathrm{ml}$ en balones de $50 \mathrm{ml}$ para obtener una concentración teórica equivalente al porcentaje estimado. Se prepara una disolución madre de acetaminofén en $\mathrm{HCl} 0.1 \mathrm{M}$, con una concentración aproximada a los $500 \mathrm{mg} / \mathrm{L}$ en un balón de $50 \mathrm{~mL}$, a partir de un patrón de pureza conocida, y se diluye $20 \mathrm{~mL}$ en $100 \mathrm{~mL}$ con $\mathrm{HCl} 0.1 \mathrm{M}$, para obtener una concentración de $100 \mathrm{mg} / \mathrm{L}$. De esta se toman alícuotas de 3 a $7 \mathrm{ml}$ en balones de $50 \mathrm{ml}$ y se aforan 
con el mismo disolvente Por último se mide la absorbancia de los patrones y las muestras de acetaminofén, en el espectrofotómetro UV con las condiciones que se muestran en el cuadro 1; y se calculan los miligramos de acetaminofén presentes, así como el porcentaje de lo etiquetado en cada caso.

Cuadro 1: Condiciones de trabajo del espectrofotómetro para todos los parámetros de la validación.

\begin{tabular}{|c|c|c|c|c|c|c|c|}
\hline Equipo & Marca & Modelo & Haz & $\lambda(\mathbf{n m})$ & Lámpara & Cubeta & $\begin{array}{l}\text { Blanco } \\
\text { reactivo }\end{array}$ \\
\hline Espectrofotóme- & Perkin & Lambda & Doble & 242 & Deuterio & Cuarzo & $\mathrm{HCl} \quad 0,1$ \\
\hline tro UV-Vis & Elmer & 20 & & & & & M \\
\hline
\end{tabular}

Para la repetibilidad y precisión intermedia se realizaron tres pesadas independientes de polvo finamente morterizado de tabletas previamente pesadas de acetaminofén, en balones de $100 \mathrm{ml}$, equivalentes a cada una de las tres concentraciones $(80,100$ y $120 \%)$, se llenan 3/4 partes del balón con HCL 0.1 M se llevan 10 minutos a baño ultrasónico, se afora con el mismo disolvente y se filtran. Posterior a esto se toman alícuotas de $1 \mathrm{ml}$ en balones de $50 \mathrm{ml}$ obteniendo concentraciones de 8, $10 \mathrm{y}$ $12 \mathrm{mg} / \mathrm{l}$. Se toman seis repeticiones de cada concentración. Se prepara una disolución madre de acetaminofén en $\mathrm{HCl} 0.1 \mathrm{M}$, con una concentración aproximada a los $500 \mathrm{mg} / \mathrm{L}$ en un balón de $50 \mathrm{~mL}$, a partir de un patrón de pureza conocida, y se diluye $5 \mathrm{~mL}$ en $50 \mathrm{~mL}$ con $\mathrm{HCl}$ $0.1 \mathrm{M}$, para obtener una concentración de $50 \mathrm{mg} / \mathrm{L}$. De esta se toman alícuotas de 3 a $7 \mathrm{ml}$ en balones de $50 \mathrm{ml}$ y se aforan con el mismo disolvente. Por último se mide la absorbancia de los patrones y las muestras de acetaminofén con las mismas especificaciones indicadas en el cuadro 1 .

Para el estudio de precisión intermedia se realizó en el mismo laboratorio, mismo analista, mismas condiciones, pero en diferentes equipos. Para esto se replica el procedimiento para repetibilidad pero se miden las respuestas en dos espectrofotomeros el "Perkin Elmer" modelo Lambda 20 y uno marca "P\&G". Se calculan los miligramos de acetaminofén presentes y el porcentaje de lo etiquetado en cada caso.

La exactitud se realizó comparando los porcentajes de recuperación con respecto al valor verdadero, independientemente de la cantidad de analito empleado en la prueba.

\section{Resultados}

A continuación se muestra en la figura 1 la superposición de las tres curvas de calibración correspondientes a las curvas realizadas para la validación del parámetro de linealidad del método, en particular todas las curvas resultaron en la misma ecuación y se obtuvo un coeficiente de correlación $\left(R^{2}\right)$ igual a 1 .

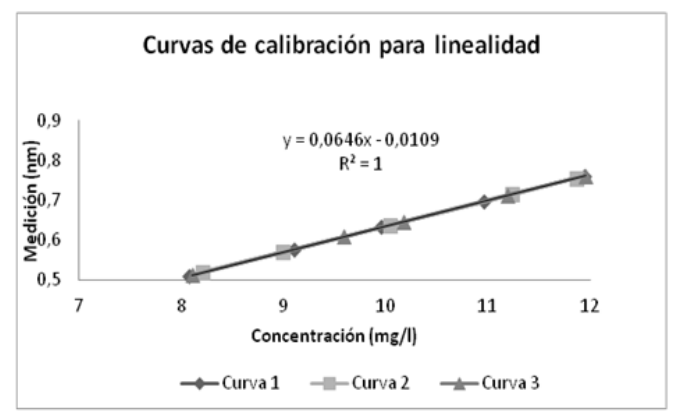


Figura 1: Superposición de curvas de calibración para comprobar linealidad.

Fuente: creación propia

En el cuadro 2 se muestran los resultados de las concentraciones obtenidas para cada nivel, de las tres curvas de calibración que comprenden la validación de la linealidad del método, y se observa gran similitud entre los resultados de las mismas.

Cuadro 2: Resultados de la validación del parámetro de linealidad del método.

\begin{tabular}{lllll}
\hline Nivel & $\%$ & Curva A & Curva B & Curva C \\
\hline 1 & 80 & 8,08 & 8,21 & 8,10 \\
2 & 90 & 9,10 & 8,98 & 9,59 \\
3 & 100 & 9,96 & 10,04 & 10,18 \\
4 & 110 & 10,95 & 11,23 & 11,19 \\
5 & 120 & 11,95 & 11,86 & 11,95 \\
Correlación (r2) & & 1 & 1 & 1 \\
Intercepto (y) & & $-0,0109$ & $-0,0109$ & $-0,0109$ \\
Pendiente (m) & & 0,0646 & 0,0646 & 0,0646 \\
\hline Fuente: creacín propia. & & & &
\end{tabular}

En cuanto a repetibilidad y precisión, en el cuadro 3 se muestra el porcentaje de lo etiquetado obtenido y una desviación estándar relativa obtenida para cada concentración y para los datos en conjunto.

Cuadro 3: Resultados de la validación del parámetro de repetibilidad del método.

\begin{tabular}{|c|c|c|c|c|}
\hline Repetición & $\mathrm{mg} / \mathrm{L}$ & $\begin{array}{l}\% \text { de lo } \\
\text { etiqueta- } \\
\text { do }\end{array}$ & DSR \% & Aceptación \\
\hline 1 & \multirow{6}{*}{8,00} & 99,10 & \multirow{6}{*}{1,30} & \multirow{6}{*}{$\leq 2 \%$} \\
\hline 2 & & 101,47 & & \\
\hline 3 & & 101,72 & & \\
\hline 4 & & 101,68 & & \\
\hline 5 & & 101,76 & & \\
\hline 6 & & 99,17 & & \\
\hline 1 & \multirow{6}{*}{10,00} & 100,65 & \multirow{6}{*}{1,52} & \multirow{6}{*}{$\leq 2 \%$} \\
\hline 2 & & 101,20 & & \\
\hline 3 & & 100,75 & & \\
\hline 4 & & 100,48 & & \\
\hline 5 & & 99,70 & & \\
\hline 6 & & 100,97 & & \\
\hline 1 & \multirow{6}{*}{12,00} & 99,80 & \multirow{6}{*}{1,44} & \multirow{6}{*}{$\leq 2 \%$} \\
\hline 2 & & 99,62 & & \\
\hline 3 & & 99,91 & & \\
\hline 4 & & 99,82 & & \\
\hline 5 & & 99,11 & & \\
\hline 6 & & 100,48 & & \\
\hline Promedio & & 100,41 & & \\
\hline DS & & 0,90 & & \\
\hline DSR \% & & 0,89 & & \\
\hline
\end{tabular}

Para evaluar la precisión intermedia se mantuvieron constantes las variables analista, día, laboratorio, y se duplicó el procedimiento de repetibilidad pero tomando la respuesta de absorbancia en dos equipos diferentes. En el cuadro 4 se muestran los resultados obtenidos por cada equipo espectrofotométrico. 
Cuadro 4: Resultados de la validación del parámetro de precisión intermedia del método.

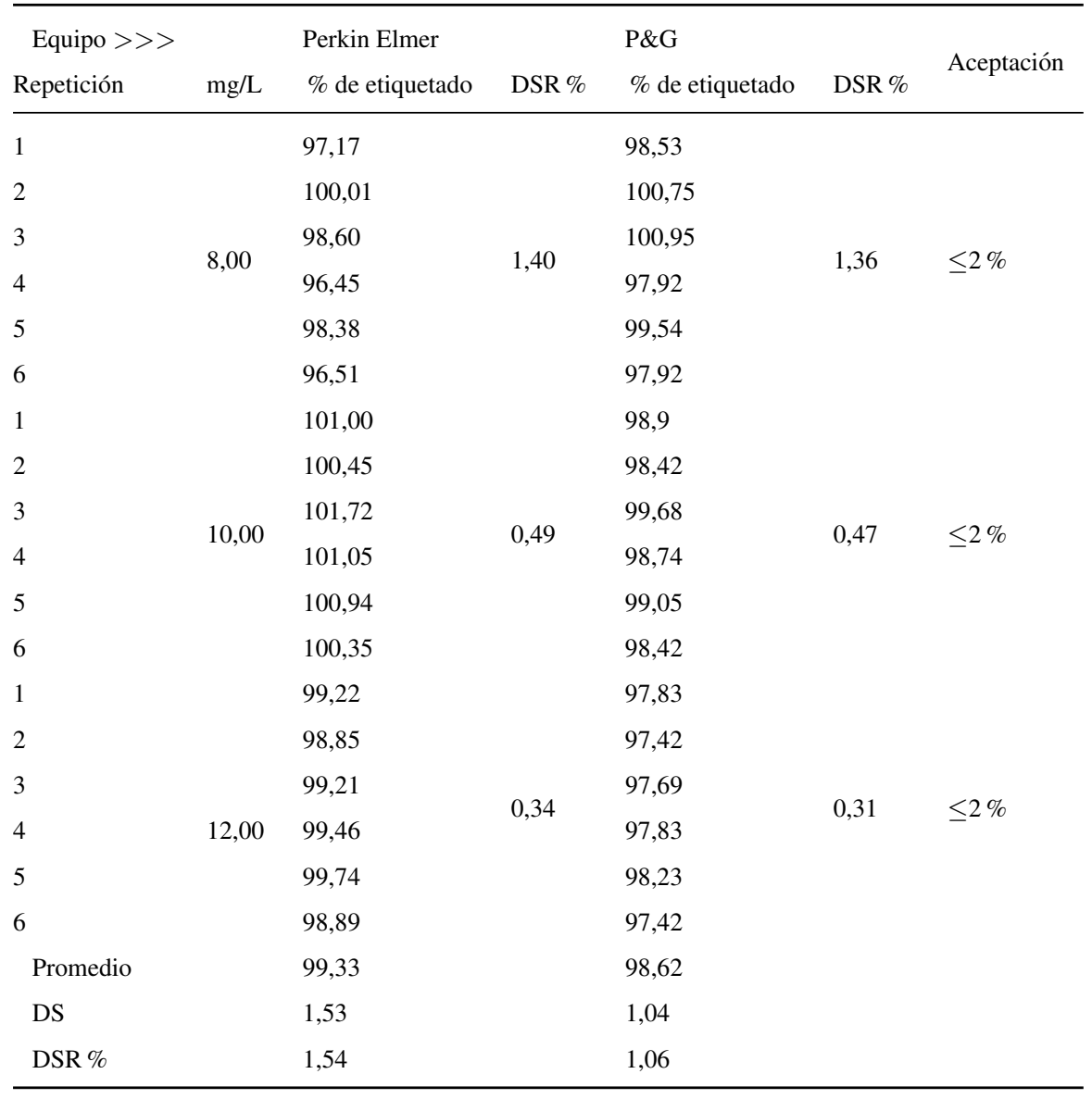

Fuente: creación propia.

Con respecto a la exactitud se estableció que ningún dato del porcentaje del etiquetado sobrepasa el parámetro de $98-102 \%$ y específicamente para métodos espectrofotométricos se permite una recuperación de 97 103 \% según establece Rodríguez (2014) para la Secretaría de Salud. Obteniendo el valor más alto de 102,26\% y el menor de 100,58\%.

En el cuadro 5 se incluyen los resultados para la exactitud del método, tomando como referencia el valor verdadero de $100,9 \%$ de lo etiquetado, obtenido para la determinación de contenido en las tabletas, analizadas por el método oficial USP por cromatografía de líquidos. 
Cuadro 5: Resultados para la exactitud del método, comparados con el valor de referencia obtenido del método oficial.

\begin{tabular}{lllll}
\hline $\begin{array}{l}\text { Conjunto } \\
\text { datos }\end{array}$ & $\begin{array}{c}\text { \% etiquetado } \\
\text { promedio }\end{array}$ & $\begin{array}{l}\text { Valor de refe- } \\
\text { rencia }(\%)\end{array}$ & $\begin{array}{c}\text { \% de recupe- } \\
\text { ración }\end{array}$ & $\begin{array}{l}\text { Parámetro de } \\
\text { aceptación }\end{array}$ \\
\hline 1 & 100,58 & & 99,68 & \\
2 & 101,28 & 100,90 & 100,38 & $97-103 \%$ \\
3 & 102,26 & & 101,35 & \\
Promedio & 101,37 & & 100,47 & \\
DSR \% & 0,83 & & 0,83 & \\
\hline
\end{tabular}

Fuente: creación propia.

\section{Discusión}

Analizando los resultados obtenidos de las evaluaciones para validación del método y según se muestra en la figura 1, correspondiente a las curvas de calibración obtenidas por triplicado a cada diferente nivel de concentración, se observa que las tres curvas arrojaron la misma ecuación de la recta y tienen una regresión de 1 , lo cual es deseable ya que según el criterio de aceptación de la Guía del Ministerio de Salud esta debe de tener un $R^{2}$ mayor a 0,995 lo cual se cumple eficientemente y existe una correlación significativa.

Debido a lo anterior, el modelo de la regresión puede explicar el $100 \%$ de la variación, consecuentemente un valor de " $y$ " puede predecir sin complicaciones una respuesta de " $x$ ", por lo tanto existe una relación estrecha entre las variables, lo cual es procedente de un comportamiento lineal.

También se demostró la homocedasticidad como criterio de linealidad, ya que para que un método sea lineal se desea un comportamiento homocedástico, es decir, similitud entre varianzas como se ilustra en la figura 2.

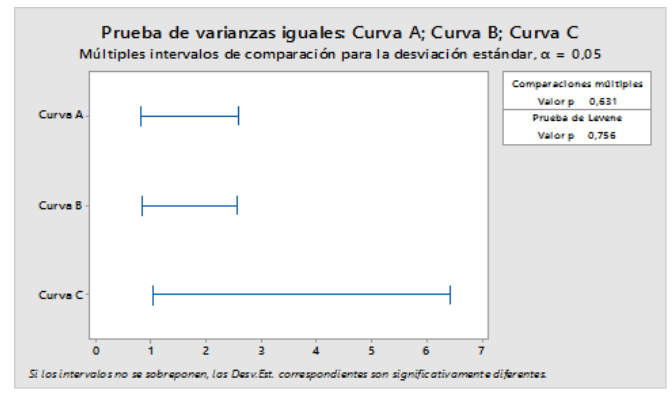

Figura 2: Análisis de Homocedasticidad. Fuente: creación propia.

Fuente: creación propia.

Según el análisis ANOVA realizado en el programa estadístico Minitab, se indicó un valor de $\mathrm{P}$ de 0,631 el cual es mayor a 0,05 por lo que no se rechaza la hipótesis nula, la cual indica que las varianzas no difieren entre sí. Se demuestra que no existe suficiente evidencia estadística que demuestre que las varianzas de las tres curvas de calibración varían entre ellas. Además, queda demostrado en la figura 2 que los intervalos se sobreponen, indicando similitud y comportamiento homocedástico, aunque la varianza de la curva tres es mayor, no se demuestra una diferencia significativa.

Además el intercepto de la ecuación de las rectas es de $b=0.0109$ la cual esta aceptablemente cerca de cero y la ecuación obtenida fue la misma para las tres 
curvas con una pendiente de 0.0646 , por lo que no hay desviación significativa ( $\mathrm{DS}=0$ ) respecto a la regresión.

Seguidamente en la figura 3, se muestra el análisis de residuos correspondiente a la validación de la linealidad del método.

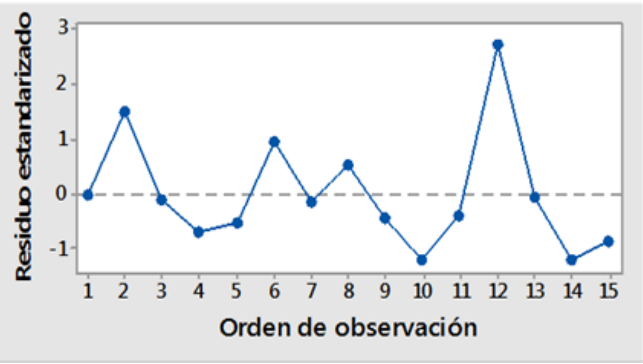

Figura 3: Análisis de residuos para linealidad. Fuente: creación propia.

Fuente: creación propia.

El comportamiento de los residuos no presenta tendencias sistemáticas, es decir, presenta un comportamiento aleatorio lo que es indicativo de linealidad, es observable que el residuo 12 es significativamente grande (mayor a 2), sin embargo, la gráfica mantiene un comportamiento satisfactorio, por lo que los residuos no están correlacionados.

Para los resultados de repetibilidad incluidos en el cuadro 3. Se observa que ningún DSR \% individual sobrepasa el parámetro establecido por el Ministerio de Salud para el contenido de principio activo el cual es de $=2 \%$ DSR ni tampoco en conjunto.

Asimismo la prueba de hipótesis realizada en comparación con el procedimiento oficial de la USP 38 por Cromatografía Líquida de Alta Resolución (HPLC), para cuantificación de acetaminofén en la prueba del “ensayo" o determinación de contenido (y de igual manera referido así para la uniformidad de contenido si tuviera que aplicarse según criterios USP); arrojó un "t" calculado de $-2,30$ contra un " $t$ ” de teórico de 2,11 por lo que no se rechaza la hipótesis nula, es decir, no existe suficiente evidencia estadística para determinar que los resultados difieren entre sí con un nivel de significancia de $\mathrm{a}=0,05$. Por lo tanto ambos métodos demostraron resultados similares y con una DSR \% aceptable para ambos métodos.

En cuanto a precisión intermedia, en el cuadro 4 se observan muy buenos desvíos relativos para los dos equipos el "Perkin Elmer" y el "P\&G", se observa que las desviaciones son muy cercanas entre si y las concentraciones son muy similares, es importante recalcar que se realizó utilizando las mismas condiciones y solo se varió el equipo, además las lecturas se realizaron el mismo día, mismo analista y de forma simultánea.

Al razonar el cuadro 5 en el resumen de resultados, para exactitud se demuestra que ningún dato del porcentaje de recuperación sobrepasa el parámetro máximo de aceptación de 97-103\%, y así se evidencia en la figura 4.

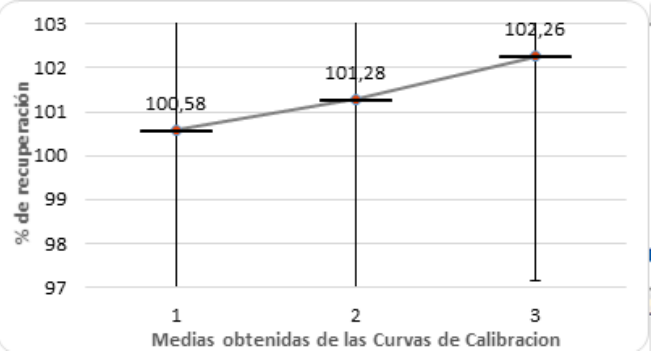

Figura 4: Porcentaje de recuperación obtenido de cada curva de calibración. Fuente: creación propia.

Fuente: creación propia.

De igual manera se comportaron los datos individuales realizados para la prueba de linealidad y posteriormente exactitud, para la cual se comparó con el procedimiento oficial (HPLC) cuyo resultado fue de $100,9 \%$ de lo etiquetado.

Por lo tanto se puede observar que el comporta- 
miento es aceptable, no obstante, en la curva de calibración 3 se presentó una tendencia mayor 102,26\%, esta no sobrepasa el límite máximo de aceptación 97-103\% de recuperación, quedando dentro de lo establecido como satisfactorio por lo que se considera que el método es exacto.

\section{Consideraciones Finales}

- Dada la importancia de contar con procedimientos estandarizados y las limitantes que se pueden topar los diferentes laboratorios de control de calidad, investigación y desarrollo, venta de servicios analíticos, u otros; son necesarias las validaciones de métodos físico-químicos, cuando estos no son oficiales, para acondicionar las metodologías analíticas y garantizar la eficiencia y eficacia en su ejecución para el fin previsto.

- Un método validado da garantía de que podrá ser

\section{Referencias}

[Avila. P, Ferro. V \& Tello. M. 1999] Avila. P, Ferro. V \& Tello. M. (1999). Estandarización de un método de análisis para analgésicos derivados de la Anilina. Consultado: 21/082015 http://www.ciencias.unal. edu.co/unciencias/data-file/farmacia/ revista/V16P39-43.pdf. (16): 39-43.

[Arias. T. 1999] Arias. T. (1999). Glosario de medicamentos. Organización Panamericana de la Salud. Recuperado de: http://www.cofepris. gob.mx/Documents/Bibliografias/ Medicamentos/Glosario.PDF.

[Durán, D. 2011] Durán, D. (2011). Análisis fisico- reproducido y utilizado con total confiabilidad bajo las condiciones en las que fue validado. Si el método requiere alguna modificación para ser utilizado por el mismo u otro laboratorio, debería ser sometido a una nueva validación para garantizar la efectividad del mismo bajo las nuevas condiciones.

- Es importante destacar que las pruebas de validación aplicadas, fueron superadas con respecto a estrictos criterios de aceptación, lo cual en comparación con el método oficial de la USP por HPLC para la determinación de contenido, u otros métodos por UV con distintas características; se obtuvieron resultados confiables y satisfactorios, y existe suficiente evidencia estadística para indicar que el método validado es adecuado, por lo que se considera lineal, preciso y exacto para la determinación de acetaminofén en la prueba de uniformidad de contenido en tabletas de $500 \mathrm{mg}$.

químicos de productos farmacéuticos en las diferentes etapas del proceso de la industria farmacéutica. Universidad de Carabobo. Facultad experimental de ciencias y tecnología. Coordinación de pasantías 1-33.

[Delfino. R, Radovancich. D \& Sarno. M. 2006] Delfino. R, Radovancich. D \& Sarno. M. (2006). Método alternativo para el control de calidad de comprimidos de paracetamol. Facultad de Ciencias Exactas y Naturales y Agrimensura. 1-5.

[Caridad. M, Garcia. P, Porto.] Caridad. M, Garcia. P, Porto. Y \& Salomon. (1999). Control de la calidad y estudio de estabilidad del paracetamol gotas orales $100 \mathrm{mg} / \mathrm{ml}$. Centro de Investigación y Desarrollo de Medicamentos. La Habana, Cuba.(28). P: 
$1-12$.

[Fernández. D, Fernández. D \& Mancipe. L. 2010]

Fernández. D, Fernández. D \& Mancipe. L. (2010). Intoxicación por acetaminofén. Revista médica de Colombia. (18)2. Recuperado de: http://www.scielo.org.co/scielo.php? pid=S0121.52562010000200008\&script $=$ sci_arttext

[Hendrickson R.G. 2015] Hendrickson R.G. (2015). Acetaminophen. In Hoffman R.S., Howland M, Lewin N.A., Nelson L.S., Goldfrank L.R. (Eds), Goldfrank's Toxicologic Emergencies, (10). Recuperado de http: //accesspharmacy. mhmedical.com. ezproxy.sibdi.ucr.ac.cr:2048/content. aspx?bookid=1163\&Sectionid=65093210.

[Lavaut, M \& Rodríguez, J. 2009] Lavaut, M \& Rodríguez, J. (2009). Validación del método de determinación de uniformidad de contenido en tabletas de dipirina de 300mg en el laboratorio farmacéutico Oriente. Revista Cubana de química 21(2): 66-69.

[Prieto. Y. 2008] Prieto. Y. (2008). Buenas prácticas de laboratorio y las normas ISO 9001:2000. Centro Nacional de Investigaciones Científicas (CNIC),
Dirección de Diagnóstico Microbiológico. Biotecnología aplicada (25). 254-257

[Rodríguez. G. 2014] Rodríguez. G. (2014). Comisión de control analítico y ampliación de cobertura. Criterios para la validación de métodos fisicoquímicos. Secretaria de Salud.1-20.

[Secretaría de Acreditación de Laboratorios. 2012] Secretaría de Acreditación de Laboratorios. (2012). Guía de validación de métodos Ente Costarricense de Acreditación (ECA). Versión: 02

[The United States Pharmacopeia Convention. 2016] The United States Pharmacopeia Convention. USP 38 - NF 33: (2016). Farmacopea de los Estados Unidos de América. Rockville, Maryland: The United States Pharmacopeial Convention.

[Informe técnico de la OMS. 2010] Informe técnico de la OMS. (2010). Anexo 1. Buenas prácticas de la OMS para laboratorios de control de calidad de productos farmacéuticos. 957. 1-48. Recuperado de: http://apps.who.int/prequal/ info_general/documents/TRS957/TRS957_ annex1_SPANISH.pdf

[Skoog, Holler \& Nieman. 2001] Skoog, Holler \& Nieman. (2001). Análisis instrumental. 5ª edición. Madrid, España: Editorial Mc Graw Hill. 